\title{
Las cegueras en la búsqueda de los conocimientos locales: el método, una deidad inconmensurable
}

\author{
Usmary Dayana Moreno ${ }^{1}$
}

Siempre existe el riesgo de construir una máquina que invente una lengua universal y si nadie comprende esa lengua, por eso mismo será aceptada por todo el mundo

Canetti

\section{Introducción}

Este ensayo es el intento de elaborar una reflexión teórica y propia a partir del proceso interpretativo que ha dado como resultado en primer lugar, pensarme como investigadora $y$, en segundo lugar, pensarme como formadora de investigadores. Mi profunda preocupación es la práctica educativa, reflexionar sobre varias interrogantes ¿Existe una forma correcta de enseñar a investigar? ¿Cuál es la forma correcta de formar investigadores? ¿De qué forma puedo construirme como investigadora de lo cualificable? ¿Cómo sería la epistemología cualitativa? ¿Existe una o varias epistemologías cualitativas? ¿Cómo superar la crisis que ha generado el construir posibilidades para lo cualitativo desde el antagonismo con la epistemología cuantitativa? Nos hemos propuesto, un grupo de profesores de la Universidad Nacional Experimental "Rafael María Baralt" (UNERMB), realizar un trabajo de investigación intitulado Programa piloto de transformación de los sistemas de investigación

$1 \quad$ Doctoranda de la Universidad de Los Andes en Educación y Antropología (Venezuela). Magister Scientiarum en Educación Abierta y a Distancia de la Universidad Nacional. Licenciada en Educación mención Dificultades de Aprendizaje. Docente e investigadora de la Universidad Nacional Experimental "Rafael María Baralt". Líneas de investigación: Estudios semióticos y culturales sobre sexualidad humana: erotismo, homosexualidad, heterosexualidad, pornografía, prostitución en Venezuela. Correo: usdamoro@gmail.com. Trujillo-Venezuela 
en las universidades venezolanas ${ }^{2}$. Un trabajo con la ambiciosa aspiración de conocer la calidad de la investigación que realiza la universidad en relación con los resultados y los beneficios esperados por y para las comunidades, pero que consideramos se convierta en aliciente para resignificar la investigación cualitativa como inacabada, en asiduidad por su libertad en el sentido que el contexto a investigar le vaya proporcionando.

Reflexionar sobre algo que a duras penas se mantiene en pie no es sencillo, la investigación cualitativa es lo que podemos considerar un feto en crecimiento, a pesar de que no es reciente su aparición. Diversas interpretaciones sobre la misma ha llevado a una práctica discursiva "acabada", "asumida" como cierta y correcta, creo para eso falta bastante y con un poco de empeño lograremos mantenerla de esa forma, porque un enfoque que intente estudiar realidades sociales, humanas, culturales, debe tener como principio lo inacabado, todas las humanas cosas son de manera contraria, son inacabadas, complejas, inciertas, misteriosas incluso. Queremos, a partir de la experiencia y de la revisión de algunos trabajos de investigación, dejar plasmada nuestra reflexión sobre la investigación y sobre la crisis que vivimos dentro de nuestras universidades para poder vincularnos con las comunidades a través de la pertinencia que tengan tanto la docencia como la investigación.

\section{La crisis de la investigación y la investigación de la crisis}

Pertenezco a una generación de profesores a quienes les ha tocado vivir una crisis profunda dentro de las universidades, debido al debilitamiento del marco teórico del cual se sirve cada universidad y cada investigador para estudiar realidades híbridas e incongruentes entre ellas, consigo mismas y, por secuencia, con los principios que rigen la vida académica. Las crisis intelectuales traen en sí dos lados, uno ostensible de la tradición científica dominante de la vida académica, del pensar y del pensamiento, y otro sedicioso, con un

$2 \quad$ Proyecto depositado en el Centro de Estudios e Investigaciones socio Económicas y Políticas CEISEP e inscrito en el CDCHT de la Universidad Nacional Experimental "Rafael María Baralt", aprobado con el código PR03-0201-CEISEP03/ SO.005-116026 en fecha 14/11/2016. 
semblante de desavenencia que causa confusiones, pero implica al mismo tiempo un reto inexcusable; la tradición científica del primer lado le hace al investigador asentir un sentido ${ }^{3}$ sosegado, la intrepidez no es una característica que signifique su práctica. El segundo lado indaga nuevas formas y aventura, por principio, sobre algo que desconoce materialmente debido a la infinidad de sentidos del cual aprehenderse para resignificar las posiciones teóricas desde las cuales concibe el mundo, es un lado expuesto, pero sin duda un lado para atisbar la condición humana de las realidades sociales y culturales.

Ambos lados de la crisis generan cierta ansiedad y producen inestabilidad en el proceso de investigación. El lado ostensible de la crisis, es el lugar previo al cambio, donde se anticipa cualquier cantidad de errores limitantes del estado actual de la investigación, cosa nada sencilla de significar. El propósito sería indagar las especificidades que caracterizan ese estado de la investigación y cuáles han sido las reglas de formación del mismo, para ello hago valer la experiencia generada durante mi permanencia en el postgrado de la Universidad Nacional Experimental Rafael María Baralt como profesora de la cátedra Tesis I de una de sus maestrías y la revisión de doce trabajos de grados generados en el mismo postgrado. La estrategia para seleccionar los mismos fue la planteada por la misma dinámica de la institución, esto es, que los mismos fueron expuestos a defensa pública y, en secuencia de aparición, fueron analizados de acuerdo a las siguientes categorías y en estricto orden:

1. El proceso de investigación: realización constructivainterpretativa del proceso, el Ser del investigador, contexto de la investigación, situación en estudio, el compromiso ontológicoepistemológico-metodológico de la investigación, forma vivencial del proceso, "robótica del proceso" (mecanización);

2. El tema de investigación (criterios de selección), el cuerpo referencial (reglas genéticas de formación), la ruta metodológica (lógica de sentido de la realidad), el análisis de datos (procesamiento contextualizado) y el producto científico (aporte o innovación);

3 Por sentido entiendo la dirección o el método, más específicamente un proceso de razonamiento para acceder al conocimiento. 
3. Respuesta científica a problemas asumidos en la investigación, articulación universidad-sociedad-producto, el maestrante y sus perspectivas en su formación como investigador (cómo realizó la investigación + cuál fue el producto + el dominio de su trabajo durante su exposición pública);

4. El proceso de enseñanza de la investigación: Ser del investigador experto (el profesor-investigador), vinculación entre lo que se enseña y lo que se hace como investigador, contribución hecha a la formación de investigadores.

La crisis, más que confusiones y debilitamientos, son oportunidades para pensarnos como investigadores y como profesores de ella, lo menciono porque en la universidad hay defensores del positivismo y de la "investigación cualitativa". Es esta la discusión más marcada, de hecho, es la única discusión, la relación entre ellos aparentemente no ha generado ganancia alguna debido al enfoque que tienen, el diálogo es apuntalarse como la mejor opción paradigmática-metódica, la que satisfaga las "necesidades" de la ciencia, los primeros y de las comunidades, los segundos. Hay una tendencia a creer que el positivismo en su espíritu positivo (organizar el desorden que había después de la revolución francesa) es la manera más "exacta" de hacer ciencia debido a la "objetividad" del proceso de investigación, por ser una forma matemática (exacta) de estudiar realidades que no se pierde entre aspectos esenciales ni simbólicos. Esto ha traído algunos problemas como la baja producción científica y la repetición de preconcepciones de problemas y de soluciones. González (2007), piensa que se debe a la fiel utilización del modelo cuantitativo (positivismo), "el positivismo que cobró vida en las ciencias sociales, y que hasta hoy continua dominando el imaginario de la investigación científica en esta área, ignoró todo lo que significa producción teórica, ideas, modelos, reflexión." (p. 1).

Agrega González (2007) que la utilización del positivismo ha afectado el proceso de investigación, pues se ha desarrollado con él un metodologismo generando con ello desagregar cualquier manifestación de una conciencia epistemológica, imponiendo "un concepto de ciencia centrado en la acumulación de datos cuantificables susceptibles de actos de verificación inmediata, sea a 
través de evidencia observacionales y/o estadísticas" (p. 1). Esto se me hizo más claro cuando los maestrantes de Tesis I me entregaban el primer capítulo de su supuesto trabajo de investigación en el primer encuentro que tuvimos. Las respuestas de los mismos ante mis interrogantes sobre cómo había sido desarrollada la investigación (por lo menos para elaborar el primer capítulo) eran inconexas, y era lógico porque ninguna de mis preguntas estaban dentro de la dinámica metódica, sino de la dinámica más o menos universal de una investigación. De diecinueve maestrantes, ninguno sabía algo más allá del método (investigación acción transformadora, IAT), estaban concentrados en realizar "paso a paso" lo que ellos consideraban era la investigación, sinónimo de método, no había la más mínima distinción de ello, y debía ser así, pues venían de un "continuo" de enseñanza basado en él. De los diecinueve, solo diez me presentaron el primer capítulo, los otros nueve se sentían "atrasados" por no tenerlo; lo primero que se evidencia en los diez trabajos es la similitud en el título, en el problema, la descripción del contexto (como todos eran IAT todos tenían las mismas "partes" en el Capítulo I), había una repetición descontrolada, todos los trabajos eran iguales, a esto es a lo que llamo robótica del proceso, pues no se produce investigación sino reafirmación de lo ya antes dicho, hay acción pero no reflexión ${ }^{4}$, y sin querer justificarlos es normal que eso suceda cuando se mal

$4 \quad$ La reflexión la asumo desde los aportes de Hannah Arendt (2007) sobre el pensar, en este trabajo asumimos que la reflexión como el pensar. Arendt (2007) nos diría "la característica principal del pensar es que interrumpe toda acción, toda actividad ordinaria, cualquiera que esta sea" (p. 165), de manera que investigar no es solo estar en contacto con realidades físicamente, es necesario darse los momentos para reflexionar sobre lo que se investiga, "El pensar siempre se ocupa de objetos que están ausentes, alejados de la directa percepción de los sentidos. Un objeto de pensamiento es siempre una re-presentación, es decir, algo o alguien que en realidad está ausente y sólo está presente a la mente que, en virtud de la imaginación, lo puede hacer presente en forma de imagen. En otras palabras, cuando pienso me muevo fuera del mundo de las apariencias, incluso si mi pensar tiene que ver con objetos ordinarios dado a los sentidos y no con objetos invisibles como, por ejemplo, conceptos o ideas, el viejo dominio del pensamiento metafísico. Para que podamos pensar en alguien, es preciso que esté alejado de nuestro sentidos; mientras permanezcamos juntos no podemos pensar en él, a pesar de que podamos recoger impresiones que posteriormente serán alimento del pensamiento; pensar en alguien que está presente implica alejarnos subrepticiamente de su compañía y actuar como si ya no estuviera" (Arendt, 2007, p. 166) 
interpretan dos cosas: a) la pertinencia del trabajo de investigación con la maestría y b) el contexto de la investigación con un contexto general.

Un trabajo, por ejemplo, que tenga como título ${ }^{5}$ La disciplina como estrategia pedagógica para la gerencia participativa, debería tener en la descripción del contexto donde se va a desarrollar un estado del arte de la disciplina en las instituciones educativas en un nivel macro, meso y micro, y cuál es la influencia que ha tenido la gerencia en ello. Ahora bien, no sucede así en el modelo de enseñanza de estas formas de investigar. Digo modelo, porque no se trata solo de estos diecinueve maestrantes, sino de todos los trabajos producidos dentro de la misma ruta. El contexto de la investigación que nos sirve de ejemplo lo desarrollarían de la siguiente forma: mencionan la ubicación geográfica de Venezuela (algunos comienzan desde la Pangea, antiguo supercontinente de la tierra), la de Trujillo, la del Plantel, cuando les corresponde hablar de la "cultura" mencionan los bailes, las comidas típicas de Carache o de Valera o de Escuque, a veces todas, y no hablan en ningún momento del estado de la disciplina como praxis, eso lo dejan para otro momento, cuando les corresponda mencionar teorías. Un maestrante que tenga la exigencia de escribir todo eso, no tiene necesidad de ir a hacer mediciones cartográficas para hacer una descripción territorial de Venezuela porque eso está hecho, tampoco iría a hacer una etnografía de la cultura de Valera o Carache porque también está hecha. Lo más inteligente es que recurran a trabajos ya elaborados (pasados por la evaluación experta de un jurado) considerados aciertos por figurar dentro del cúmulo de productos "reales" aceptados por una comunidad científica, yo también lo haría. Ante la exigencia la obediencia, y más si de eso depende el título de magister. El otro asunto es cuáles son los criterios de selección del tema a investigar.

La IAT busca transformar realidades incongruentes, y con bastante ayuda no ha podido lograrse porque esa transformación

5 En este caso no develo el título ni el contenido real del trabajo de ninguno de los maestrantes porque para la fecha de esta publicación aún no han sido presentados en defensa pública, pero si recreo un posible modelo en el cual están sumergidos los trabajos de grado y la forma de enseñar a investigar. 
suena a utopía más que a un cambio real de conciencia, hay que establecer la distinción entre el pensamiento científico y el mítico ${ }^{6}$ cuando se realiza este tipo de investigación y cualquier otra que busque incorporar a lo investigados como investigadores. La forma de seleccionar el tema ${ }^{7}$ es el inicio del problema. Un investigador que asista a una comunidad en pro de ella a resolver problemas a través de la "reflexión" para transformarla frente a la selección del tema (jerarquización) participativa corre el riesgo de dejarse llevar por la "mayoría", es decir, si en la comunidad existen problemas de violencia y drogas, por ejemplo, y la mayoría dice que eso es porque no hay una cancha y no hay iglesia y el investigador (en formación en caso de los maestrantes) accede a ello y busca ayuda para construir una cancha y una iglesia, pero no transforma el problema en solución, sino lo profundiza, estaríamos provocando un serio problema como universidad y como visionarios en la formación de investigadores comprometidos con la sociedad venezolana. Porque lo que debe suceder allí es que el investigador, si no es experto en el tema de la violencia y las drogas indague, por lo mínimo, y logre ver que eso ya se ha hecho antes y no ha dado resultado, ni las iglesias ni las canchas resuelven esos problemas y un científico lo debe saber, mientras que el mito recurriría a "Dios" para resolverlo, la ciencia recurriría a la

$6 \quad$ El pensamiento mítico para Cassirer (1998, p. 95) "coloca todo lo real en el mismo plano, una misma sustancia, no "tiene" distintos atributos, sino que cada atributo en cuanto tal ya es sustancia; esto significa que esto solo puede aprehenderse en concreción inmediata", la pragmática, o sea, sin reflexión.

$7 \quad$ Participación activa de todos los sujetos investigadores-investigados juntos, en este punto, Fals Borda consideraba investigadores a los miembros de las comunidades, así que el trabajo de detección, análisis y reflexión deben hacerlo entre todos, y hasta ahí vamos bien; lo que sucedió después (la proliferación deshonesta de una investigación bastante aproximada en un principio a lo social-cultural, terminara siendo un apéndice del positivismo pero con otro nombre) es que le legaron el trabajo científico a personas que se encuentra mentalmente fuera de la ciencia y muy dentro del pensamiento mítico, a esta forma de investigar se le debieron considerar no solo los aportes de la sociología también los de la antropología. El mito para Cassirer (1998) "solo sabe lo inmediatamente existente y operante. De allí que las relaciones que establece no sean conexiones con el pensamiento a través de las cuales se enlace y diferencie al mismo tiempo lo que entra en ellas, sino que constituyen una especie de pegamento capaz de aglutinar de algún modo hasta las cosas más heterogéneas" (p. 93), y para despertar la conciencia hace falta salir del mito por leves momentos aunque sea. 
incursión de la lógica en la que se formaron esos problemas, la ciencia no es mito y si la sangre de los teólogos (como refiere metafóricamente Nietzsche) siguen dominando el pensamiento científico vamos a estar perennemente en esta crisis. Porque no es el método el que soluciona, son los investigadores, a través de un proceso de investigación que incluye método, episteme, teorías, conocidas y por conocer, lo que puede mantenernos en una praxis real investigativa. La oportunidad que nos da una investigación como la IAT de generar conocimiento nuevo a través de la valoración de lo real se está viendo afectada por la tradición científica en la que aún nos encontramos como universidad, pero con posibles salidas desde lo individual, es decir, que los investigadores que logran hacer lectura del trasfondo en el que está metida la IAT logra darle tal vez a ella o a cualquier otra la relevancia que pueda tener.

Ahora bien, existen otras consecuencias de la selección del tema, si la disciplina es lo que desea investigar, es de suponer que existe disciplina en la institución para querer estudiarla y dar aportes a esa comunidad científica, pero los maestrantes ante la interrogante de por qué seleccionó el tema (en vista de que el contexto está incorrectamente desarrollado) refieren que en su escuela hay mucha violencia, entonces las cosas cambian, un investigador experto (profesor de la cátedra) debe inmediatamente darse cuenta de que el maestrante está en un error, si no hay disciplina y si, en cambio violencia, lo que va a investigar es la violencia no la disciplina, porque no se puede investigar lo que no hay, a menos que quieras resignificar el término disciplina. La selección del tema depende de muchas cosas, pero en principio de lo que le apasione al investigador, porque eso generará productos científicos exitosos, aunque suene romántico, la pasión es la que mueve al sujeto en la vida y en la razón (Apolíneo y Dionisos).

La IAT se ha constituido en una "rutinaria" manera de hacer las cosas o ésta se ha desvirtuado con el incorrecto desarrollo o ella no ha sido suficiente para poder alcanzar una "investigación cualitativa" que nos lleve a superar la crisis. Con el positivismo se impone un método, un tema, una teoría y una solución, la realidad hay que encuadrarla dentro de ello, esta metodología... 
...condujo a un metodologismo, donde los instrumentos y las técnicas se emanciparon de las representaciones teóricas, y se convirtieron en principios absolutos de legitimidad para la información producida por ellos, cuyo procesamiento no pasaba por la reflexividad de los investigadores (González, 2007, p. 2),

Esto es totalmente cierto, y tanto que me hace recordar una experiencia que tuve durante unas conferencias que se desarrollaban en la universidad ${ }^{8}$ donde una ponente, defensora del positivismo, ante mi interrogante sobre sus afirmaciones acerca del "aporte" que investigaciones de este tipo dejaban a las escuelas, "el instrumento"; la investigadora refería ante dos interrogantes que le haría sobre sus "aportes", uno era el riesgo que se corría al utilizar un cuestionario como un generador de datos infalible en vista de que tanto la realidad como las teorías son movibles y, dos, si ella estaba consciente de que en nuestras escuelas persisten serios problemas en vista de que los docentes no investigan y reflexionan sobre su práctica para generar conocimientos propios y no ser tan dependientes de lo "ajeno" y que con seguridad ese "aporte instrumental" causaría más daño que bienestar y su respuesta fue concisa y sencilla, "no porque el instrumento que yo elaboro con mis estudiantes es muy amplio y abarca muchas cosas y además las teorías no avanzan tanto".

Aunque desconcertante la respuesta en cierta forma deja ver la inocencia de una práctica investigativa que no lleva a solucionar problemas y en cambio sí a la comercialización académica debido a la irreflexividad donde entran los investigadores en formación, a veces me suele rondar la pregunta ¿será intencional?, ya esa sería otra investigación. Esta práctica está basada en lo que González (2007) llamó instrumentalismo:

El instrumentalismo ha llevado a una hegemonía al proceso de recolección de información en las ciencias sociales. Los instrumentos en esta tradición han sido

$8 \quad$ A propósito de los 34 años de la creación de la UNERMB, tantos años en la misma forma, la esperanza es no llegar a los 234 años haciendo lo mismo, estamos a tiempo. 
asociados a categorías universales de significación, a través de las cuales se establecen relaciones directas y universales entre ciertos significados y formas concretas de expresión del sujeto, con lo cual su aplicación no pasa de una rutina clasificatoria. El instrumento es utilizado como criterio de afirmación conclusivo, con lo cual los procesos de investigación, evaluación y diagnostico que los utilizan, no pasan de ser procesos clasificatorios en los que el investigador procura aplicar un conjunto de conocimientos preestablecidos más que producirlos ( $\mathrm{p}$. 2).

El instrumentalismo le ha seguido el juego a la anhelada búsqueda de objetividad, generando con ello una crisis profunda en la producción de conocimiento, sobre todo del local, que es el que ha estado subordinado a los aspectos universales de los enfoques académicos. La IAT es lo inverso y, lastimosamente, no se ha logrado provocar la reflexividad de los investigadores-investigados; en el afán de buscar la reflexión se ha caído en la confusión de unir el pensamiento científico con el mítico. El instrumentalismo al que se está conduciendo la IAT como único método para hacer "investigación cualitativa" es salir de una cárcel del pensamiento para entrar a otra. Esto es porque existen muchas otras formas (espero que sean millones) de hacer investigación del modo que supere la dualidad binaria en la que está sumergido nuestro imaginario, existen tantas epistemes como culturas, cada una tiene su forma de conocer y relacionarse con el mundo, no tiene por qué existir una sola porque seguiríamos en la crisis y no me refiero a una paradigmática, sino a una de modos de enfocarse desde la libertad que debe gozar todo investigador. Los paradigmas no son opciones viables para esta libertad, el mismo Kuhn lo estableció, solo que causó una confusión con ello.

Kuhn (1992) diría entre muchas otras cosas que "los paradigmas obliga a los científicos a investigar alguna parte de la naturaleza de una manera tan detallada y profunda que sería inimaginable en otras condiciones" (p. 53), se entiende que un positivista se ate a este tipo de investigación, a esta obligación, pero ipor qué lo haría un investigador de la acción transformadora, donde se supone que su 
principio es abrir posibilidades de conocimientos, saberes, diálogo de saberes, participación activa y reflexiva, crítica y todo lo demás que implique saber y conocimiento local? A este tipo de investigación Kuhn (1992) la denominó ciencia normal "realización lograda mediante la ampliación del conocimiento de aquellos hechos que el paradigma muestra como particularmente reveladores, aumentando la extensión del acoplamiento entre esos hechos y las predicciones del paradigma y por medio de la articulación ulterior del paradigma mismo" (p. 52), con certeza se puede afirmar que el positivismo corresponde a esa ciencia normal, investigar siempre sobre los mismos conocimientos, razón por la cual "un paradigma es raramente un objeto para la renovación" (Kuhn, 1992, p. 51) porque tiene un espíritu universal, es un marco de interpretación que delimita cualquier intento de hacer distintas las cosas, pero no nos damos cuenta cómo lo hace (más adelante demostraremos como), de manera que si el paradigma es sinónimo de universalidad, quiere decir que no solo el método lo es, también la teoría, la forma de concebir la realidad, los problemas e incluso las soluciones.

Los doce trabajos revisados ( 7 positivistas y 5 IAT) tienen exactamente la misma dinámica, ninguna de ellos genera un aporte al conocimiento establecido, repiten lo ya antes dicho e investigado, los títulos son casi idénticos, los referentes no son construidos reflexivamente sino copiados de otros trabajos, los resultados aburren por no generar algo novedoso, que ponga en cuestión lo ya conocido y que nos rete en cierta forma a cambiar argumentos, las recomendaciones son las mismas, los positivistas y los IAT recomiendan lo mismo, pero lo más grave es que esta última apuesta a la trasformación y así se declara, transformadora, cuando en realidad, si hablamos con honestidad es repetidora, solo que colectivamente. En todas las "investigaciones" hechas con el método IAT utilizan algo que llaman paradigma sociocrítico y el tipo de investigación IAT, es una mezcla extraña. Cómo alguien que dice valorar los conocimiento locales, lo cual implica respetar la lógica de sentido (método) generada de la misma realidad y de la situación a resolver, utiliza el "paradigma sociocrítico", no sé claramente la insistencia, porque el mismo creador de la palabra la utilizaría para significar una práctica incoherente con 
la investigación, con una ciencia revolucionaria, así lo manifestó, la lógica propia estaría opuesta a identificarse en un universal, estaría dispuesta sí, a trabajar conjuntamente pero no a subsumirse a ella, el paradigma implica conocimiento general la investigación para que algún día logre llamarse cualitativa (algo que dudo si seguimos en este camino) debe suspender la utilización de lentes universales para ver cosas locales.

Imaginemos por un momento un antropólogo haciendo etnografía o etnología y que antes de ir al campo se ponga "la camisa" del método y de acuerdo a este es que vaya haciendo su trabajo y que esta camisa le predefina la ruta a seguir y que además lo haga encuadrar la realidad del pueblo de Samoa con el de los Waraos, por ejemplo, es un absurdo. Pues eso hace un paradigma encuadrar, amarrar, limitar, atar, cegar al investigador sobre aspectos propios y lo lleva por un camino. Nos manejamos en un metodocentrismo a la hora de hacer investigación y nos estamos debilitando en el intento de rescatarnos de la ceguera donde nos puso el positivismo, el racionalismo y el empirismo.

Pero preguntémonos que fue lo que hizo que Kuhn significara el paradigma, él observaba en el siglo pasado, que esta forma de hacer ciencia tenía dos características notables, los seguidores eran pocos debido a los escasos referentes para apoyar la práctica científica, y por ende el limitado grupo de científicos no podían abarcar mayor cantidad de problemas, dejándolos sin resolver. Toda práctica científica que poseía esas dos características las denominó Kuhn, paradigma. ¿Tiene ambas características la IAT?, la respuesta es afirmativa, las tiene. Porque el grupo de investigadores que hace esto no es muy grande (además de no hacerla correctamente) y porque deja un montón de problemas sin resolver ya que no sabe cómo hacerlo; recurren en el error de no hacer la distinción entre el pensamiento mítico y el científico. Las universidades se han encargado de mantener esta práctica, así cuando se está en un lugar metodológico, la epistemología no es precisamente un "recurso necesario", Arendt (2007) manifestó que en tiempos de dictadura era más fácil actuar que pensar, y cuando se impone una forma de ver, de leer, de aprender, de indagar y de conocer se está dentro de una. 
He llamado crisis intelectual a la crisis que vivimos en las universidades y en la forma de hacer ciencia porque la práctica científica está sumida completamente a la categoría de continuidad irreflexiva aunque ahora justamente debo hacer lo que hace la tradición, ir a los orígenes. El ensayo escrito por Kuhn (1992) produce algunas molestias en la cultura de la ciencia debido a la influencia tan marcada que aún tenemos de él y de los significados de ciencia normal y de paradigma. Existe un uso exacerbado de ellos, lo cual ha sido el impedimento primario para que hoy podamos hablar de investigación cualitativa. Ante la prerrogativa de reflexionar sobre lo cualitativo, hay que expresar lo evidente, la investigación cualitativa no existe. No existe porque sus cimientos carecen de versatilidad $y$, en cambio, adolecen de la generalización, la dualidad binaria y el antagonismo; la investigación cualitativa no existe porque se ha construido como el antagónico de lo cuantitativo, provocando un camino inverso que la lleva al mismo lugar; en la intención de alejarse de la "investigación cuantitativa" hemos caído en el ciclo del eterno retorno. Lógicamente si no existe la investigación cualitativa, tampoco la cuantitativa, aunque contemos con técnicas de recolección y análisis de datos cualitativos y cuantitativos, pero eso sería una fase del proceso de investigación no toda. Existe -en retórica al menos- la intención y la fuerza de alejarse del positivismo, pero hasta ahora solo desaciertos hemos encontrado en el proceso.

Hay un problema aún más serio y creo debe pensarse. Quise pretensiosamente hacer una ruptura de esa dinámica que vengo mencionando, la cual consistió un reto de inmensurables alcances, a mi parecer, o por lo menos amplio. Con estudiantes de pregrado de otra universidad, había puesto en práctica una forma de enseñar a investigar, mi forma (no en el sentido de que sea pionera, no lo sé, en el sentido de hacerlo yo de esa manera y tratar de tener una vinculación entre lo que enseño y lo que hago) y quise experimentarla con los estudiantes de postgrado donde se hace necesaria aún más aprender a investigar. El problema que presentan los trabajos de investigación es la confusión que existe entre el informe de investigación y el proceso de investigación. Cuando regreso en el tiempo a mis días de formación, recuerdo esta traumática experiencia de tener que "hacer 
el primer capítulo", una exigencia que implicaba competencias, es decir, conjunto de habilidades y destrezas que no tienes cuando estás en formación, se tienen habilidades y destrezas por separados pero juntas no; no es lo mismo decir que tengo habilidades para la indagación o la lectura y la escritura, que tener la competencia de indagar, leer, escribir, calcular entre otras, juntas actuando en un proceso de investigación.

Para construir el "primer capítulo" debe a ese punto haberse realizado un arqueo bibliohemerográfico que haya dotado al investigador de conocimientos sobre el tema seleccionado, haber leído teorías diversas que den una amplitud acerca de lo que se ha dicho del tema, indagar sobre investigaciones más recientes para ver hasta dónde es pertinente el estudio propio, de donde partieron los otros, la ruta utilizada y los resultados logrados, para que no se vaya a hacer una investigación repetida ${ }^{9}$ y en cambio utilizar los aportes para desarrollar los propios. Cuando ya se tiene ubicado todo esto, corresponde desmontar cada teoría y cada estudio reciente y, a manera de interpretación, descargarla todo en un diario de campo (cualquier cuaderno) con citas claro está, pero con reflexiones propias sobre esa teoría y sobre los datos empíricos que se tienen. Un profesor que enseñe a investigar y, lógicamente, a pensar, debe saber hacerlo él mismo, para que la amplitud con la que se hable frente a los estudiantes sea tan enriquecedora que se pueda fomentar una formación de investigadores conexa con la sociedad en la cual está integrada la universidad. También, antes de hacer "el primer capítulo" se debe establecer en ese diario de campo la ruta metodológica a seguir.

¿Qué sucede si se pide el primer capítulo sin enseñar antes al maestrante a hacer el arqueo bibliohemerográfico? que el informe

9 Los antecedentes de una investigación, bajo cualquier método, son tratados como un ornamento, los buscan y los agregan al trabajo sin interpretación alguna del proceso hermenéutico que implica citar un trabajo de investigación de otra persona, simplemente "parafrasean" la relación "posible" y a veces imposible de este estudio con la investigación en desarrollo y listo, no se vuelven a mirar los antecedentes. Los antecedentes pueden servir para el producto teórico por ejemplo y hasta ahora no he visto a más nadie hacerlo, lo cual lamento porque sería interesante tener esa amplitud y triangular formas de construir los conocimientos. 
de investigación pasa a ser un instrumento de la investigación y, por lo tanto, impondría una forma universal de escribir y "por pasos" inconexos, así que alguien que haga el "primer capítulo" está muy lejos aún de saber que ruta metodológica va a utilizar y cuales argumentos teóricos utilizará para su trabajo. Por eso es que cuando un investigador (novel) se encuentra frente al "marco teórico" produce un estado de crisis propia, y como le ha sido enseñado por el experto que las teorías "existen" y solo deben "parafrasearlas" se ha generado una estructura tan rígida de este "capítulo" que termina siendo una copia fiel y exacta de los trabajos anteriores, porque si solo deben "parafrasear" ¿para qué van a interpretarla? Y para qué va a tomarse la molestia (la dura molestia) de leer las fuentes, si ya otros trabajos lo tienen, lo más fácil es ir y copiar y asumir como suyas esos trabajos, que al final ninguno es puro, todos son copias de copias. Lo más grave no es la copia exacta, lo más grave es que los maestrantes cuando caen en el eterno retorno de entregar los capítulos a sus profesores para que los corrijan, se desesperan tanto que terminan en la comercialización descarada y deshonesta que existe, el mercado de la fabricación de trabajos de grado (fabricación de sujetos irreflexivos,) está en ascenso, mientras que nuestra capacidad intelectual y académica disminuye proporcionadamente.

Como bien dicen aquellos que tienen como primera opción de conocer lo sistémico, el mismo sistema ha creado tanto el plagio como la comercialización del intelecto, pero esto constituye otras rutas que desarrollaremos más adelante en nuestro camino de valorar cuál es la calidad de la producción de nuestra universidad y de la universidad venezolana. Los estudiosos de los métodos y los procesos de investigación se encuentran en un punto: no hay producción científica dentro de las ciencias sociales que satisfagan las necesidades sociales, culturales y humanas.

Realidades complejas no pueden abordarse con un método exclusivo, porque las realidades no se agotan, por tanto todos tienen algo que decir de ella. La matemática al igual que la hermenéutica, las historias de vida, la etnografía, la etnología, la investigación acción, la investigación acción participativa, la semiótica, la etnometodología, el interaccionismo simbólico, el positivismo entre otros, nos pueden 
dotar de miradas diversas para hacer lecturas de lo local y son además imprescindibles para ello, la perspectiva correcta es asumir que sobre realidades locales se pueden hacer abordajes con enfoque matemáticos y cualitativos. Cada uno de estos métodos tienen sentidos diferentes, pero todos ellos, con excepción de las matemáticas, pueden hacernos llegar a argumentos que no por no ser matemáticos dejan de ser científicos. Lo "cualitativo", si es que algún día logramos consolidar algo así, no puede construirse en opuesto a lo cuantitativo, allí está el error. Satanizar la matemática y en cambio sobrevalorar las "cualidades", eso no nos ubica en el territorio de lo cualitativo, sino en el territorio de la utopía y ahí sí salimos de la ciencia.

La salida de la crisis no es algo que se pueda delimitar en tiempo, espacio y sentidos, en cambio se hace el motivo idóneo para aligerar el pensar y el pensamiento y lograr a través de ellos, posicionarse brillantemente al centro del problema, conocerlo y poder establecer o alcanzar conclusiones sobre él en un nivel lógico, esto es, que el sentido de la argumentación permita reorganizar en términos de las mismas realidades las condiciones de su existencia. Creo como Kuhn (1992) que "las crisis son una condición previa y necesaria para el nacimiento de nuevas teorías" (p. 128) y también una forma de romper la continuidad en la que se encuentran formas tradicionales de producir conocimientos científicos, no quiere decir, sin embargo, que las ya agotadas formas quedan fuera de funcionamiento porque los investigadores aun teniendo plena consciencia de lo débil que se hicieron esas formas de indagar el mundo, no renuncian fácilmente, la continuidad irreflexiva del sentido tiene como ya antes nos diría Foucault (2007) "todo un juego de nociones que diversifican, cada una a su modo, el tema de la continuidad" (p. 33) aprovechándose de nociones como la tradición, la influencia, desarrollo, evolución, mentalidad y espíritu. Si existe alguna forma sencilla de entender lo que es un paradigma, es comprendiendo los elementos que actúan dentro de esta continuidad.

Estas nociones se subsumen a la categoría continuidad irreflexiva de Foucault (2007) y son utilizadas como principio regulador de "las cosas" dentro de un discurso, de tal manera, la tradición frente a lo desemejante activa el estado "original" como único referente para 
mantener en vigilia la inmodificación discursiva, nos dirá el filósofo que la noción de tradición "autoriza a reducir la diferencia propia de todo comienzo, para remontar sin interrupción en la asignación indefinida del origen" (p. 33) apoyada por la influencia la cual sirve de fundamento a los proceso de transmisión y comunicación y "que refiere a un proceso de índole causal los fenómenos de semejanza o de repetición; que liga a distancia y a través del tiempo, a unidades definidas como individuos, obras, nociones o teorías" (p. 34). Ambas nociones hacen las veces de un poderhabiente del científico y de la práctica científica, de manera que cuando se está frente a la tradición científica, se está frente a un poder inconmensurable del cual no es fácil desapegarse, habría que hacer como dice Foucault un trabajo negativo, el cual tampoco muestra caminos sencillos de llevarse a cabo, para muestra tenemos la utilización de métodos dentro de las universidades como la investigación acción participativa, la etnografía y todo lo que pueda declararse como "investigación cualitativa", los cuales terminan siendo incorrectamente desarrollados pues en el afán de querer distanciarse de las formas matemáticas de estudiar realidades terminan haciendo lo mismo pero de forma invertida; es decir, forma unisentido que se centra en el sujeto como antagonismo del objeto, en las emociones y afectividades opuesto a la objetivación, en las "cualidades" en vez de las "cantidades".

El trabajo negativo que sugiere el filósofo, refiere al rompimiento de esa forma de hacer ciencia, la necesidad de la investigación cualitativa no debe oponerse al positivismo (filosofía positiva propuesta por Comte), sino poner a disposición formas otras de conocer, desagregándonos de la confusión en la cual nos ha sumergido el florecimiento de paradigmas. La tradición y la influencia nos mantienen en el eterno retorno de investigar como ya antes lo hicieron otros, a veces pienso en que fue lo que le permitió a Einstein crear sus teorías, y de seguro fue la NO utilización de paradigmas para crear categorías nuevas que nos hicieran comprender mejor el mundo y todo lo que se encuentra fuera de él. Es irónico decirle a un estudiante piense si quien lo enseña no lo hace.

El científico durante las crisis hace lo que la tradición le indica, reorganizar todo de acuerdo al referente original, el cual lo sustenta 
teórica y epistémicamente para poder comprender lo sucedido y darse respuestas dentro del torbellino de "verdades" que lo apañan. Esto lo logra aprehendiéndose de las nociones desarrollo y evolución, las cuales "permiten reagrupar una sucesión de acontecimientos dispersos. Referirlos a un mismo y único principio organizador, someterlos al poder ejemplar de la vida" (Foucault, 2007, p. 34), la vida ejemplar es la vivida antes de la crisis, la que estaba dotada de todas las respuestas necesarias para comprender las cosas "más importantes", del sentido inexcusable, quien metodológicamente proporciona la única ruta que sabe transitar, partir siempre de la misma forma, resolver los mismos problemas y llegar al sitio de confianza y seguridad científica. Abandonar todo esto, no es cuestión de toma de decisión sino de clarificación del desorden que nos ha causado la crisis.

Se debe añadir a esta categoría de continuidad irreflexiva la noción de mentalidad o espíritu, esta noción, en mi opinión, es la más difícil de detectar, no es sólida pero se siente y se vive, hace que fenómenos de épocas distintas se comuniquen a través de "lazos simbólicos, un juego de semejanza y de espejo, o que hacen surgir como principio de unidad y de explicación la soberanía de una conciencia colectiva" (Foucault, 2007, p. 34), esos lazos simbólicos implica una comunicación simbólica creadora de significados compartidos entre sujetos de la misma tradición, por consecuencia los científicos de la decadencia tendrían que ejercer un pensamiento de quiebre de la costumbre resultado de esta práctica intelectual continua.

Estamos obligados a pensar esta crisis en el mismo juego de su instancia, ¿qué la provocó? Y ¿cómo es que no logramos salir de ella? Al paradigma hay que ponerlo en jaque. Kuhn (1992) en esta crítica a la forma de hacer ciencia en los años sesenta también la denominó tradicional, puesto que cuando surgen situaciones problemas no comprensibles los referentes científicos no son precisamente los más adecuados para afrontarlas, así que la vía no es resignificar el sentido y pensar unos nuevos, sino ajustar estos problemas de manera que puedan ser abordables por los métodos ya aceptados por esta comunidad. El soporte a esta tradición ha sido durante mucho tiempo la influencia que ha tenido el proceso educativo en mantener 
la misma forma "segura" de "investigar", a través de dispositivos o fenómenos de semejanza o de repetición (Foucault, 2007), como han sido los libros de ciencia y, por qué no mencionarlo, como han sido los textos de metodología. "Esos libros científicos exponen la teoría aceptada, ilustran todas o muchas de sus aplicaciones apropiadas y comparan éstas con experimentos y observaciones de condición ejemplar" (Kuhn, 1992, p. 33).

Una representación sobre la investigación cualitativa es la concerniente al sentido que esta utiliza; existen diferentes tendencias, una de estas es la asignación del método inductivo como propio y "natural" en ella, algo que resulta inverosímil desde una epistemología que tiene como condición la libertad del sentido con dominio autónomo y, además, hay quienes manifiestan que el método del positivismo es el deductivo, pero hay investigaciones positivistas de tipo experimental que utilizan la inducción, esto nos debe llevar a reflexionar sobre lo que estamos haciendo para superar la importancia que le damos al método y la poca que le damos al tema, debemos entender que no es el método el que soluciona los problemas, sino los sujetos, el método es el sujeto. Aunque no es fácil entenderlo, nuestra práctica de investigación está seriamente afectada por la técnica, es lo que podemos encontrar cuando nos ubicamos en una línea del tiempo, dónde puede que tenga sus raíces todo esto, ciertamente Descartes (1637) es bastante influyente, pero a quienes debemos premiar por ser los detonantes es a Platón y a Sócrates. Platón (2005), privilegió la mente sobre el cuerpo, de manera que la poesía y el arte no debían ser consideradas necesarias, solo la razón y el amor a la sabiduría, de manera que la verdad era lo que había de buscarse a través del método que Platón consideraba útil la crítica, no el sentir, de tal manera que la crítica responde a la razón y nos da la verdad y las artes, los sentimientos y las emociones a la vida, al cuerpo y como éste nos hace "pecar" hay que alejarlo todo lo que se pueda para estar dentro de "los sabios" y de la ciencia, no es una locura cuando Nietzsche (1983) considerara esta filosofía decadente, las ideas de un consenso que lleva a subordinar un sujeto a lo universal, en el caso del cristianismo a un dios, en el caso de la ciencia al método, de manera que si seguimos entendiendo la investigación como método, 
estaremos en la lógica teológica (el lugar donde viven los mitos más antiguos), cambiamos a Dios por el Método y despojamos de cualquier intento de reflexividad a los científicos sociales. Así que esto aplica tanto para el positivismo como para cualquier otra forma de investigar que intente establecerse como una deidad.

\section{Reflexiones finales}

Parto del principio cada cosa que existe en el mundo ha sido producto de la actividad humana, entendiendo como cosa todo aquello a lo que se le puede aplicar un sentido, una dirección y además puede ser sometido siempre a procesos de significación y resignificación, porque pertenecen a la vida social y cultural. Así, desde esta perspectiva ponemos a circular como ejemplo de cosa la biología. La biología es la ciencia que estudia la vida en el planeta, es una ciencia "natural" generadora de grandes conocimientos sobre los seres vivos y la cual sirve de apoyo a muchas otras ciencias, entre estas, las llamadas sociales y humanas. La pedagogía es una de ellas. La pedagogía utiliza los aportes de la biología y con ellos reflexiona sobre su objeto de estudio, la educación. Así que cuando un pedagogo se activa en reflexión, lo hace preconcibiendo que existe ante todo un ser biológico, luego psicológico, social y si se tiene suerte un sujeto cultural, y con los conocimientos científicos que se tienen se puede "modificar" o "transformar" realidades sociales "complejas" que no alcanzan muchas veces la comprensión humana, la biología dota de objetividad las argumentaciones sobre lo humano y despeja dudas al respecto. Esto como ejemplo es una de las consecuencias a las que nos ha traído este metodocentrismo.

Este trabajo es apenas el primero de tres publicaciones que se harán en torno al tema de la investigación y el estado del arte de la misma, haciendo una especie de hermenéutica que me permita comprender el proceso de la investigación desde otra concepción menos rígida, además de seguir reflexionando sobre la vinculación que debe tener la docencia y la investigación. Romper tradiciones no es algo sencillo, pero afortunadamente hay quienes ya en compromiso consigo, primeramente, buscan las mejores formas de hacerlo. 
La crisis nos ha llevado a la instauración de una ciencia compiladora y repetidora encargada de mantener vigente teorías por periodos de tiempos indeterminados, problemas y soluciones predefinidas y el uso del método científico (como método universal de obligado uso para todo aquel que busque hacer ciencia) para llevar a cabo la práctica de conocer por toda una comunidad científica, una ciencia enraizada en la tradición y el universalismo del conocimiento, desmereciéndole créditos a la posibilidad de existencia de conocimientos y problemas locales no relacionados con él; lo local responde a sus propias reglas de existencia, por lo tanto en los conocimientos generales no se encuentra identidad. Nada nuevo, ya antes Kuhn (1992) lo habría expuesto cuando describiría el espíritu de continuidad en el cual se constituía la ciencia que se hacía dentro de los paradigmas, dentro de los cuales se producía la verdad aceptada por los miembros de una comunidad científica, de esta manera todos los científicos partidarios de un paradigma investigan siempre de la misma forma, con el mismo método y los mismos problemas a solucionar.

Valorar lo propio implica entender que existen conocimientos y saberes en lugares varios, por lo cual es correcto afirmar la existencia de conocimientos locales nacidos de lógicas particulares con métodos propios y los cuales responden a la lógica de sentido de cada realidad y no necesariamente tenga relación con conocimientos globales; existe la creencia que un científico lo es siempre y cuando utilice el método científico para llegar a la verdad de las cosas, afirmación cierta tal vez para aquellos que se encuentra dentro de las ciencias naturales, pero los miembros de las ciencias sociales y humanas entran en conflicto cuando deben estudiar problemas humanos y sociales, el método científico es un método universal del cual hay que desprenderse para poder tener acceso a los conocimientos locales y producir un quiebre en la ceguera que causa la ciencia en esta perspectiva. Si las realidades son cambiantes, debe aplicar el mismo principio en el uso de teorías y de métodos. Pero la experiencia es otra.

La experiencia nos dice que las realidades son cambiantes, pero los métodos y las teorías son estáticos, el motivo podemos ubicarlo en la ausencia de la razón epistemológica (o conciencia epistemológica 
como manifestara González) y el uso exacerbado del método científico, del metodocentrismo en el cual se ubica la práctica de la investigación y la enseñanza de la misma. La epistemología nos permite estudiar las lógicas del conocer, de los sentidos, de los significados de la ciencia y su pertinencia con los contextos de acuerdo al momento histórico donde se enuncian. La actividad del conocer se ha construido como una práctica de continuidad irreflexiva, los llamamientos que hacen algunos investigadores a reflexionar sobre las realidades y contextos propios ni son gratis ni son irracionales, es la única salida a la superación de la crisis en la que estamos sumergidos; la culpa del uso indiscriminado del eurocentrismo no es de los europeos, es nuestra, porque ese llamado a mirarnos, a conocernos en lo propio no puede hacerse ni imponiendo métodos ni mucho menos reglamentos, nadie trae a la consciencia algo bajo coacción. ¿Qué es lo que caracteriza esa forma universal de hacer ciencia? La utilización del método científico ¿Qué es lo que nos impide salir de la crisis? Lo mismo, la utilización de la investigación acción participativa como única forma de conocer lo local.

\section{Referencias}

Arendt, H. (2007). Responsabilidad y Juicio. Barcelona: Ediciones Paidós Ibérica.

Descartes, R. (1637). Discours de la me'thode: Übers und hrsg von Lüder Gäbe. Hamburg: Felix.

Cassirer, E. (1998). Filosofia de las Formas Simbólicas. III: fenomenología del reconocimiento. Trad. Armando Morones. México, D.F: Fondo de Cultura.

Foucault, M. (2007). La arqueología del saber. Madrid: Siglo XXI Editores.

González, F. (2007). Investigación cualitativa y subjetividad, los procesos de construcción de la información. México, D.F: McGraw-Hill.

Kuhn, T. (1992). La estructura de las revoluciones científicas. México, D.F: Fondo de Cultura Económica.

Nietzsche, F. (1983). El anticristo. Madrid: Alianza Editorial Madrid. Platón (2005). Diálogos, Tomo I. Bogotá: Ediciones Universales. 